\title{
Reactivation of an old memory during sleep and wakefulness
}

\author{
BERNARD HARS and ELIZABETH HENNEVIN \\ Laboratoire de Physiologie Nerveuse, C.N.R.S., Gif-sur-Yvette, France
}

\begin{abstract}
In a series of four experiments, the effects of the reactivation of an old memory in different states of vigilance were investigated. Rats were trained to run in a six-unit spatial discrimination maze for food reward with low-level electrical stimulation delivered to the ear when they entered blind alleys. Twenty-five days after training, electrotactile stimulation was given to animals as a reactivating treatment just before a retention test. When the cuing treatment was given during wakefulness, retention performance was improved. When it was given in slow-wave sleep, it had no effect. When it was given in paradoxical sleep, performance was poorer. The use of control groups permitted an assessment of the specificity of the cuing effect in each case: Pretest presentation of the same stimulus did not modify performance when it had no cue value. Together, these results show that the effect of the reactivation of an old memory depends upon the vigilance state in which reactivation takes place. They suggest that reorganization of an old memory could be accomplished during paradoxical sleep.
\end{abstract}

There has been a long-standing interest in the role of sleep in memory function, and, indeed, the beneficial effect of sleep on the retention of information acquired during wakefulness was demonstrated long ago in humans (Heine, 1914; Jenkins \& Dallenbach, 1924; Van Ormer, 1932). More recent studies, mostly in rodents, have provided substantial evidence for a relationship between postlearning paradoxical sleep (PS) and memory processes (for reviews, see Fishbein \& Gutwein, 1977; Hennevin \& Hars, 1985; Hennevin \& Leconte, 1977; McGrath \& Cohen, 1978; Pearlman, 1979; Smith, 1985). First, postlearning PS deprivation was shown to impair performance in a variety of complex learning tasks. Moreover, an increase in the duration of PS was observed during the period of sleep occurring spontaneously after learning. Most recently, such a postlearning PS elevation was also observed in humans (Mandai, Guerrien, Sockeel, Dujardin, \& Leconte, 1989). And finally, we have shown that a newly formed memory trace can be modulated during postlearning PS (Hars, Hennevin, \& Pasques, 1985; Hennevin, Hars, \& Bloch, 1989). For example, we have found that retention of a new memory can be enhanced by giving a nonawakening reminder during PS phases following learning. All these data led us to propose that a new memory could be reprocessed during postlearning PS. Through this reprocessing, PS would serve to promote the assimilation and integration of new experiences.

A theoretical problem lies in the fact that reasoning in experimental studies is most often based on the premise

\footnotetext{
We wish to thank Michèle Dumas for her helpful technical assistance. Requests for reprints should be addressed to Bernard Hars, Département de Psychophysiologie, LPN2, CNRS, 91198 Gif-sur-Yvette cedex, France.
}

that a new memory trace is elaborated in a tabula rasa neuronal network. It is more than likely that the elaboration of a new memory implies some form of reorganization of already existing memory programs. Such a reprogramming process could, opportunely, take place during sleep, when the organism is relatively protected from interfering inputs. One of the contributions of sleep to retention could be that it facilitates the reorganization of old memory programs. From this proposition emerges the underlying assumption that old memory traces can be reprocessed during sleep along with newly acquired memory traces. However, although relations between sleep and newly formed memories have been thoroughly explored, the relationship between sleep and old memories has, as yet, not been investigated. So, until now, the hypothesis that an old memory can be recycled during sleep has had no experimental support. An important first step in support of this hypothesis would be to demonstrate that reactivation of an old memory can take place during sleep.

In alluding to the reactivation notion, we refer to the active-inactive memory distinction made by Lewis (1969, 1979). A memory trace is in an active state when the characteristics it presents are the same as those of a justcreated memory: both are subject to similar rules of strengthening and weakening, and both are susceptible to further elaboration, as opposed to memories in an inactive state.

A large variety of data coming from experiments performed in awake subjects have demonstrated that a memory might enter into the active state when a cuing treatment is delivered. Empirically, a cuing procedure consists of exposing the subject to some component of the original learning situation without submitting it to a complete learning trial. Through this procedure, it has been shown that the retrieval of a memory can be modi- 
fied by the pretest presentation of a cue related to the target memory. Moreover, cuing has been shown to render a previously stored memory trace as sensitive to the effects of various agents, either facilitative or disruptive, as is the case for memory shortly following learning (for a review, see Spear, 1973, 1978).

The cuing procedure has been widely used in a variety of paradigms, and it still appears to be effective at very long delays after learning (Dekeyne, Deweer, \& Sara, 1987; Deweer, Sara, \& Hars, 1980; Gatti, Pais, \& Weeks, 1975; Gisquet-Verrier, Dekeyne, \& Alexinsky, 1989). Thus, it is possible for cuing to reactivate even a longestablished memory. Although almost all experiments using a cuing procedure have heretofore been performed during wakefulness, we have succeeded, in two attempts, in introducing a cue related to a newly formed memory in both PS and slow-wave sleep (Hars \& Hennevin, 1987; Hars et al., 1985). Indeed, we have shown that when the stimulus used as a CS during avoidance conditioning was given as a reminder in postlearning sleep, subsequent retention performance was modified. No effect was detected when the stimulus had not been previously associated with the learning task.

Thus, cuing could offer an opportunity to test the possibility of reactivating an old memory during sleep. The aim of the present experiments was to test this possibility by studying the effects of pretest cuing given during different states of vigilance (wakefulness, slow-wave sleep, and paradoxical sleep) after a long retention delay.

\section{GENERAL METHODS}

\footnotetext{
Animals

Naive male Sprague-Dawley rats, obtained from Iffa Credo, SaintGermain sur l'Arbresle, France, served as subjects. They weighed $220 \mathrm{~g}$ on arrival in the laboratory. For the duration of the experiment, they were housed in the colony room in individual cages and were maintained under a 12-h light-dark cycle $(8$ a.m.-8 p.m. light). One week before pretraining, they were placed on a fooddeprivation schedule in which the daily ration, given between 6 and 7 p.m., was gradually reduced to $10 \mathrm{~g}$ over 5 days. This regimen was maintained (with some individual corrections when necessary) throughout the pretraining and training periods. Under these conditions, the animals weighed approximately $85 \%$ of their freefeeding weights at the end of the training period.

Surgery

All the animals were implanted with three cortical and two neckmuscle electrodes. Silver extradural cortical electrodes (ECoG) were placed along the interhemispheric suture with a large frontoparietal contralateral derivation. This permits recording of hippocampal theta activity (Timo-Iaria et al., 1970). Two silver electrodes were sewn through the dorsal neck muscles for the recording of electromyographic (EMG) activity. The electrodes were connected to the pins of a miniature socket fixed to the skull with acrylic cement. A double earring, made with two looped silver wires $(0.4 \mathrm{~mm}$ in diameter, $2 \mathrm{~cm}$ in length) fixed to a light plug $(7 \times 5 \times 3 \mathrm{~mm})$, was permanently hooked to one ear. This bipolar stimulation electrode was used to apply mild electrotactile stimulation. The surgery was performed under general anesthesia (pentobarbital, $60 \mathrm{mg} / \mathrm{kg}$, i.p.) 3 weeks before the beginning of the experiment.
}

\section{Apparatus}

The linear maze consisted of six opaque Armodur $\mathrm{T}$ units $(50 \times 40 \times 35 \mathrm{~cm}$ each) with $10-\mathrm{cm}$ openings between them. A $25 \times 10 \times 35 \mathrm{~cm}$ startbox was separated from the first maze unit by a sliding door. The goalbox $(25 \times 25 \times 35 \mathrm{~cm})$ could also be closed by a sliding door. The choice point, or stem of the $T$, was located $15 \mathrm{~cm}$ from the entrance to each unit. The transverse of the $T$ was $25 \mathrm{~cm}$ from the choice point. The barriers in each unit of the maze could be changed from one side to the other. The maze configuration was LRRLLR or RLLRRL. The entire maze measured approximately $3 \mathrm{~m}$ in length. The top of the maze was open. A 3-m-long rail was placed $1 \mathrm{~m}$ above the maze. The stimulating cable connected to the ear of the rat was hung with rubberbands from a hook that could slide on the rail. This arrangement did not disturb spontaneous maze-exploration behavior. The maze was located in a room (the experimental room) adjacent to the colony room.

\section{Electrotactile Stimulation}

During the pretraining period, the intensity of earshock subsequently used during training was determined for each animal. In the experimental room, in a cage similar to the home cage, the behavioral responses to electric shocks to the ear were observed in each rat as it was awake and immobile. The electrotactile stimulation (ETS) consisted of a square wave of 250-msec duration; the intensities were varied and delivered in a random order. The chosen intensity induced a slight, but clear and reproducible, head movement, with no flinch or other reaction that might suggest that the animal had felt pain.

\section{Design and Procedure}

Pretraining period. The first 5 days constituted a pretraining period during which the animals were familiarized with the experimenter, the maze, and the experimental conditions. On Day 1, each rat was allowed to feed for a 20 -min period in a box that was similar to the goalbox of the maze and located in the experimental room. The food reward consisted of broken nuts. On Day 2, the rats, in groups of 6 , were given $10 \mathrm{~min}$ to explore the last maze unit and the goalbox, with the sliding door open, the barrier removed, and broken nuts available in the goalbox. On Days 3 and 4 , always using the last maze unit, individual rats were trained to enter the goalbox. After the rat entered the goalbox, the sliding door was closed and the rat was allowed to feed for $2 \mathrm{~min}$. On Day 4, each animal was trained with a cable connected to the ear. The procedure was the same on Day 5, but the entire maze with the barriers removed was used.

Training. Training began on Day 8 . The barriers were placed at the appropriate points, and the path was changed to its mirrorimage configuration for every other rat. Each animal was carried from the colony room to the experimental room and placed into the startbox with a stimulating cable connected to the ear. The sliding door was then immediately opened, and the time it took for the rat to reach the goalbox was recorded, as was the number of incorrect choices (errors). It was possible to record multiple errors in one maze unit if the rat returned to the preceding maze unit or units and retraced an incorrect choice. For animals in the stimulated conditions, each time the rat entered a blind alley (rear paws beyond the stem of the T), it received an ETS on the ear for $250 \mathrm{msec}$; this was repeated every second for as long as it stayed in the blind alley. ETS intensity was that previously assessed for each individual; it could be slightly modified according to responsiveness. Animals in the nonstimulated conditions received no stimulation in the maze. All animals were allowed to eat in the goalbox for $2 \mathrm{~min}$ before being removed and returned to the colony room. There were five training trials, with a 24-h intertrial interval.

At the end of training, separately for rats in the stimulated and in the nonstimulated conditions, the animals were divided into two 
matched groups. The groups were equalized on the basis of mean performance on the last two trials (Acquisition Trials 4 and 5). The performance criteria used were both total time to run the maze and number of errors.

Training-to-test interval. The animals were kept in their home cages with access to food and water ad lib for the first part of the training-to-test interval. Seven days before retention testing, the gradual reduction in food was reintroduced, just as before pretraining. During this week, recording and stimulating cables were connected to each animal in its home cage for $3 \mathrm{~h}$ each day. This familiarization procedure was performed in order to (1) habituate the rats to sleep while being connected to cables, and (2) decrease the probability that the connecting in itself would later serve as an effective reminder cue.

Retention test. On the test day, 25 days after the last training trial, recording and ear-stimulating cables were connected to each animal of each group in its home cage. The "cued" rats received ETS in their home cages just before testing. ETSs were similar to those used during training, except for the intensity, which was weaker during this pretest period for two reasons. First, reactivity to ear stimulation was higher when the awake animal was quiet in its home cage than when it was running in the maze. Second, it was necessary to use an intensity that did not induce awakening when delivered in pretest sleep. After this treatment, the rats were carried to the experimental room and placed into the startbox of the maze with a stimulating cable connected to the ear. The retention test was similar to a learning trial. The "noncued" rats received no ETS before testing. They were submitted to the test trial in the same conditions as the other group. As during training, the exact path traced by each rat, its errors, and its total time to run the maze were noted.

\section{EXPERIMENT 1}

To use a cuing procedure during sleep, several problems must be resolved. The cue must be effective outside the experimental context; it should allow for its introduction during sleep without awakening the animal; its physical parameters should be easy to control; and it must not be perceived by animals in neighboring cages.

A pilot study was performed to find an experimental procedure that met these criteria. Previously, we had shown that retrieval of memory of a maze after a long retention interval could be facilitated by cuing just before testing (Deweer et al., 1980). However, the cuing treatment used was an exposure to background stimuli in the experimental room, and that, of course, could not be used during sleep. We therefore modified the learning situation by giving slight ETSs on the ear when the rats entered a blind alley of the maze. After the 25-day trainingto-test interval, ETS was used as a cue and given in the home cage before testing. A comparison of the performances of the experimental (cued before testing) and control (not cued) animals showed that the cued rats made significantly fewer errors $[F(1,24)=4.47, p<.05]$. Control animals made more errors on the test trial than on the last acquisition trial $[F(1,13)=8.57, p<.025]$. By contrast, no forgetting could be detected in the cued animals, which maintained the same level of performance $[F(1,11)=2.13$, n.s. $]$.

The aim of the present experiment was to replicate the findings of this preliminary study. Control groups were added in the experimental design, in order to ensure that the improvement in performance observed after pretest cuing was actually due to the cue value of earshocks and not to some nonspecific proactive effect of earshocks on subsequent performances. So we studied the effect of pretest earshocks when they had not been associated previously with the learning task. Thus, the purpose of this experiment was twofold: (1) to demonstrate the necessity for the pretest stimulus to be associated with original learning in order to affect retention performances, and (2) to confirm that exposure to an intratask stimulus can reactivate an old memory trace, even outside of the training context.

\section{Experimental Plan and Procedure}

Subjects. Forty-six rats served as subjects.

Training. After pretraining, the animals were divided randomly into two groups:

1. The stimulated rats (Group $S, n=24$ ) learned the maze with ETS delivered in blind alleys. The mean intensity of behavioral responsiveness to ETS assessed before training was $162 \mu \mathrm{A}$ (range: 120 to $185 \mu \mathrm{A})$.

2. The nonstimulated rats (Group NS, $n=22$ ) were not given ETS in the maze, although behavioral responsiveness to ETS had been assessed before training. Mean intensity was $159 \mu \mathrm{A}$ (130 to $180 \mu \mathrm{A})$. During the training and testing, a cable was connected to the ear of the animals even though they did not receive ETS.

Retention test. At the end of training, each group was divided into two matched subgroups on the basis of mean performance on the last two trials.

On the test day, just prior to retention testing:

1. The stimulated-cued rats (Subgroup $S-C, n=12$ ) received ETS in their home cages. ETS intensity was $25 \%$ below the intensity used in the blind alleys during training. Five 250 -msec ETSs were delivered in a 60 -sec period. Then $30 \mathrm{sec}$ were allowed to elapse before the animal was taken to the experimental room.

2. The stimulated-noncued rats (Subgroup S-NC, $n=12$ ) received no ETS.

3. The nonstimulated-cued rats (Subgroup NS-C, $n=10$ ) received five ETSs in the same conditions as did the $S-C$ rats.

4. The nonstimulated-noncued rats (Subgroup NS-NC, $n=12$ ) received no ETS.

For each subgroup, the test trial was exactly the same as a learning trial (i.e., with or without ETS delivered in the blind alleys of the maze).

This experimental design is illustrated in Figure 1.

\section{Results and Discussion}

The animals showed considerable improvement in performance during training (see learning curves for run times and errors in Figure 2). Delivery of ETS during trials did not modify the rate of acquisition. There is no difference between the learning curves of Groups $S$ and NS, regardless of acquisition index used. Moreover, delivery of ETS in the maze had no apparent effect on retention performance. Subgroups NS-NC and S-NC displayed similar performance at testing $[F(1,22)<1$ for both running times and errors).

Considering retention performance, one subgroupSubgroup S-C - contrasts with that of the other three. Indeed, these animals performed as well at testing as they had at the end of training. A within-group comparison 
PRE-LEARNING

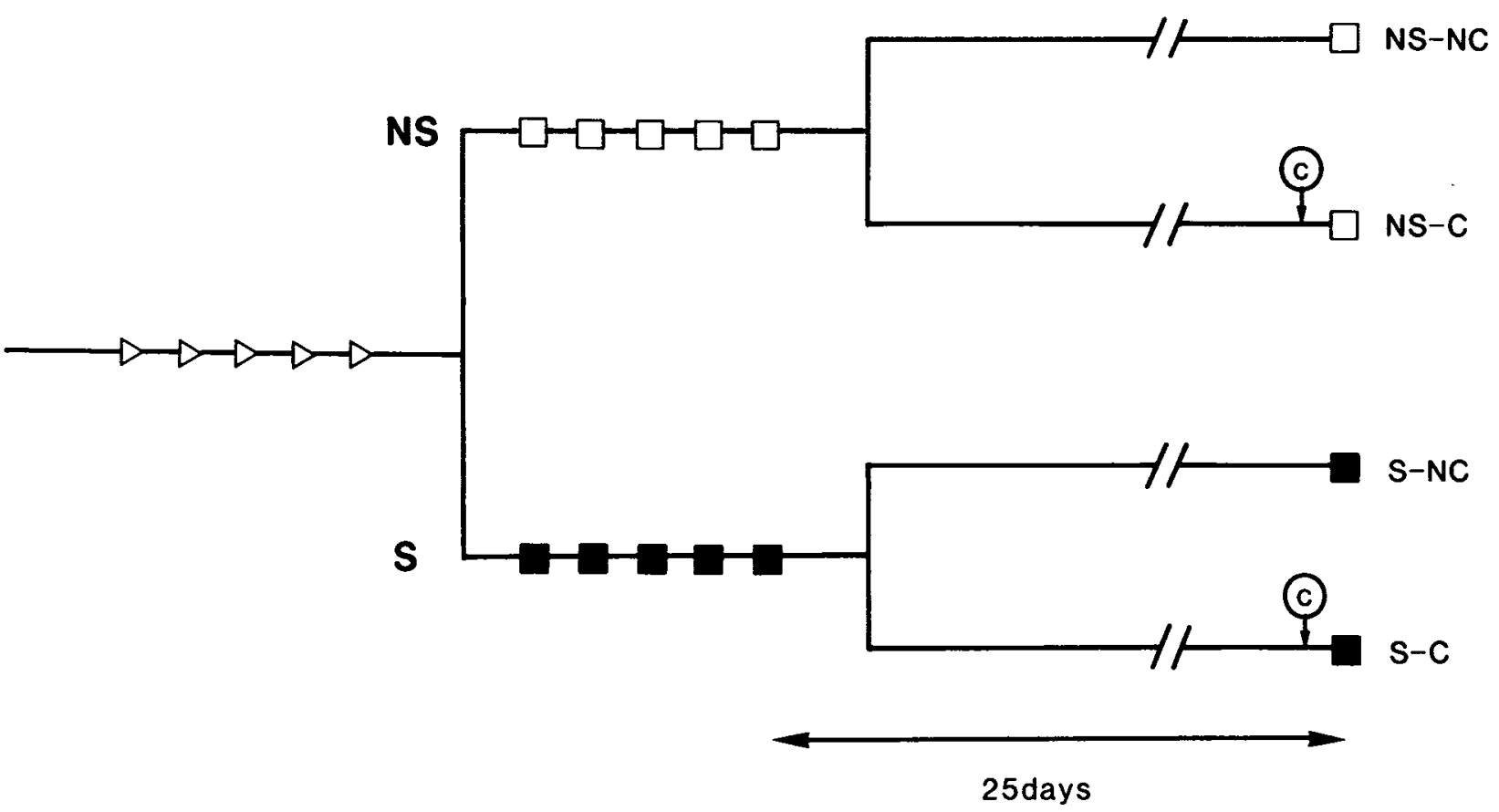

Figure 1. Basic experimental design used in this series of experiments. After a prelearning period of 5 days, five training trials were performed with a $24 \mathrm{~h}$ intertrial interval. A retention test took place 25 days after the end of training. In Experiment 1 , Group $S$ learned the maze with slight electrotactile stimulations on the ear (ETS), delivered when the rats entered blind alleys. Group NS did not receive ETS during training. Just prior to testing, Subgroups S-C and NS-C received ETS in their home cages during wakefulness. Subgroups S-NC and NS-NC received no ETS before testing. $S=$ stimulated (i.e., ETS in blind alleys of the maze), $N S=$ nonstimulated, $C=$ cued (i.e., ETS before testing), and NC = noncued.

of error scores showed no significant difference between the last acquisition trial and the test trial $[F(1,11)<1)$. The animals ran more slowly at testing, but the difference was not significant $[F(1,11)=4.55$, n.s. $]$. By contrast, the animals in the other three subgroups showed marked forgetting, that is, a performance decrement over time. In the retention test, they took more time to run the maze [for Subgroups S-NC, NS-NC, and NS-C, respectively: $F(1,11)=8.03, p<.025, F(1,11)=15.99$, $p<.005$, and $F(1,9)=10.30, p<.01]$ and made more errors $[F(1,11)=7.17, p<.025, F(1,11)=5.08$, $p<.05$, and $F(1,9)=6.68, p<.05]$ than they had at the end of acquisition.

Overall analysis of variance showed significant differences among the four groups for errors $[F(3,42)=2.84$, $p<.05]$. Between-groups analyses revealed the superiority of the animals that had been both stimulated and reminded (Subgroup S-C). First, a comparison between the subgroups that had received ETS during learning showed that the animals that were cued before testing (S-C) performed significantly better than noncued animals $(\mathrm{S}-\mathrm{NC})$ in terms of errors $[F(1,22)=4.82, p<.05]$. Second, of the animals that received ETS before testing, those in Subgroup S-C showed better performance than did those in Subgroup NS-C in terms of errors $[F(1,20)=7.18, p<.025]$. The inefficacy of pretest earshocks when they are meaningless is confirmed when the two subgroups that were trained without earshock in blind alleys are compared. Indeed, NS-C and NS-NC animals displayed very similar performance at testing $[F(1,20)<1$ for errors]. For running times, no statistical differences appeared among the groups $[F(3,42)<1]$.

Two major points can be drawn from these results: (1) They corroborate the results of previous experiments (Deweer \& Sara, 1984; Deweer et al., 1980) by showing that spontaneous forgetting resulting from a long training-to-test interval can be alleviated by pretest cuing. Furthermore, they demonstrate that an isolated intratask cue given in the home cage is as effective as background stimuli. (2) They indicate that the cue is effective in modulating retrieval only when it is an element of the initial learning task. When the pretest stimulus has not been associated with learning, it has no effect on subsequent performance. This rules out an interpretation in terms of nonspecific effects of the pretest treatment. In the same way, it is important to emphasize the fact that the effects of cuing do not apply to run times, which should be the performance index preferentially affected 

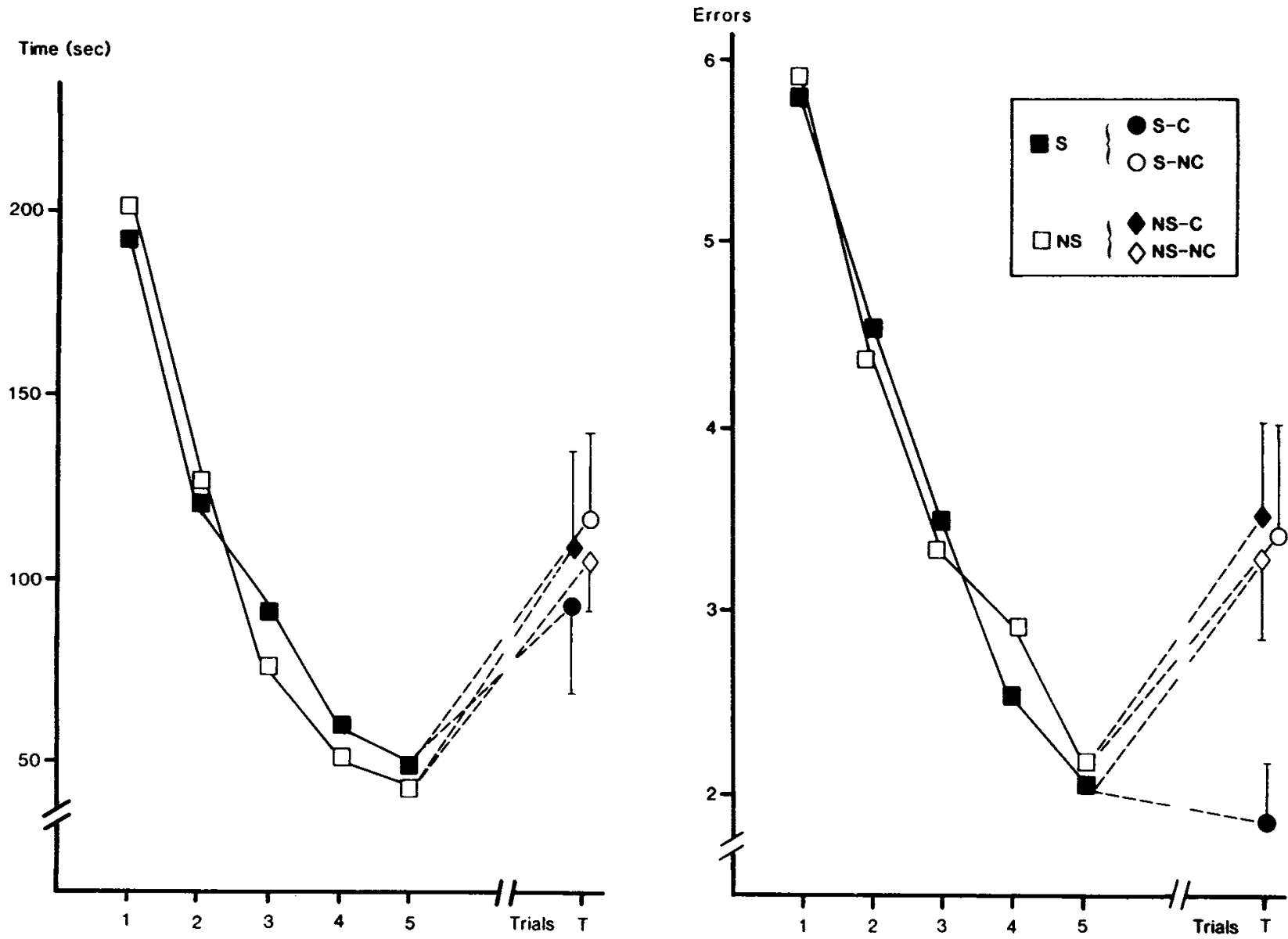

Figure 2. Mean time to run the maze (on the left) and mean number of errors (on the right) for the two groups during training trials and for the four subgroups at retention test $( \pm S E M) 25$ days later. Group $S$ received ETS during training; Group NS did not. Subgroups $C$ received ETS during a period of wakefulness just prior to testing; Subgroups NC did not. Note (1) the lack of effect of ETS on the learning curves, and (2) the deficit in performance as measured by errors at testing in all subgroups, except Subgroup S-C, which received ETS both during training and before testing.

by some nonspecific influences (such as locomotor activity, arousal, motivation). They are limited to errors that can be considered to reflect the most cognitive aspect of performance in this task. These data are similar to those of previous studies which used the same maze task (e.g., Dekeyne et al., 1987): pretest cuing after a long trainingto-test interval facilitated performance in terms of errors only. Thus, in this experimental paradigm, the more effective memory retrieval in cued animals is reflected by their more accurate discrimination.

\section{EXPERIMENT 2}

This experiment was designed to test the possibility of reactivating an old memory trace during slow-wave sleep (SWS). The experimental design was basically the same as that of Experiment 1, except that all the animals were submitted to the retention test immediately after the end of an SWS phase.

\section{Experimental Plan and Procedure}

Thirty-two rats were used as subjects. They were housed, handled, and food deprived as in the preceding experiment. Mean intensity of ETS behavioral thresholds determined before training was $153 \mu \mathrm{A}(105$ to $180 \mu \mathrm{A})$. All the animals learned the maze with weak ETS applied to the ear when they entered a blind alley.

The retention test took place 25 days after the end of training. ECoG and EMG activity was polygraphically monitored so that vigilance states could be recognized as they occurred. All animals were submitted to the retention test after spontaneous awakening from an SWS phase. They were divided into two groups, matched according to their performance on the last two training trials:

1. The cued rats (Group SWS-C, $n=16$ ) received ETS in their home cages during an SWS phase. ETSs were delivered at least $1 \mathrm{~min}$ after the beginning of SWS. Their intensities were varied. Intensity was weak for the first ETS and then progressively increased, but it was never strong enough to be awakening. The mean ETS intensity was $23 \%$ lower than it was during maze learning. On average, 9.5 ETSs were delivered in about $120 \mathrm{sec}$ during pretest SWS.

2. The noncued rats (Group SWS-NC, $n=16$ ) received no ETS during the SWS phase that preceded the test trial.

Immediately after spontaneous awakening, the rats were carried from the colony room to the experimental room. They were con- 
nected to the stimulation cable and placed into the startbox of the maze.

\section{Results and Discussion}

The animals showed good learning over the five training trials (see the learning curves in Figure 3).

Delivery of ETS during SWS did not alter SWS. Mean duration of SWS preceding the test trial did not differ in cued $(178 \mathrm{sec})$ and noncued $(182 \mathrm{sec})$ animals.

At testing, as shown in Figure 3, all the animals showed forgetting. Cued and noncued rats took longer to run the maze and made more errors on the test trial than they had on the last acquisition trial. Within-group comparisons indicated significant increases for Groups SWS-C and SWS-NC both in errors [respectively, $F(1,15)=6.20$, $p<.025$, and $F(1,15)=8.22, p<.01]$ and in running times $[F(1,15)=11.5, p<.005$, and $F(1,15)=10.43$, $p<.01]$. Moreover, all the animals displayed the same amount of forgetting. At testing, no significant difference could be detected between the performance of the two groups $[F(1,30)<1$, for both errors and running times].

Thus, the cuing treatment had no effect when applied during pretest SWS.

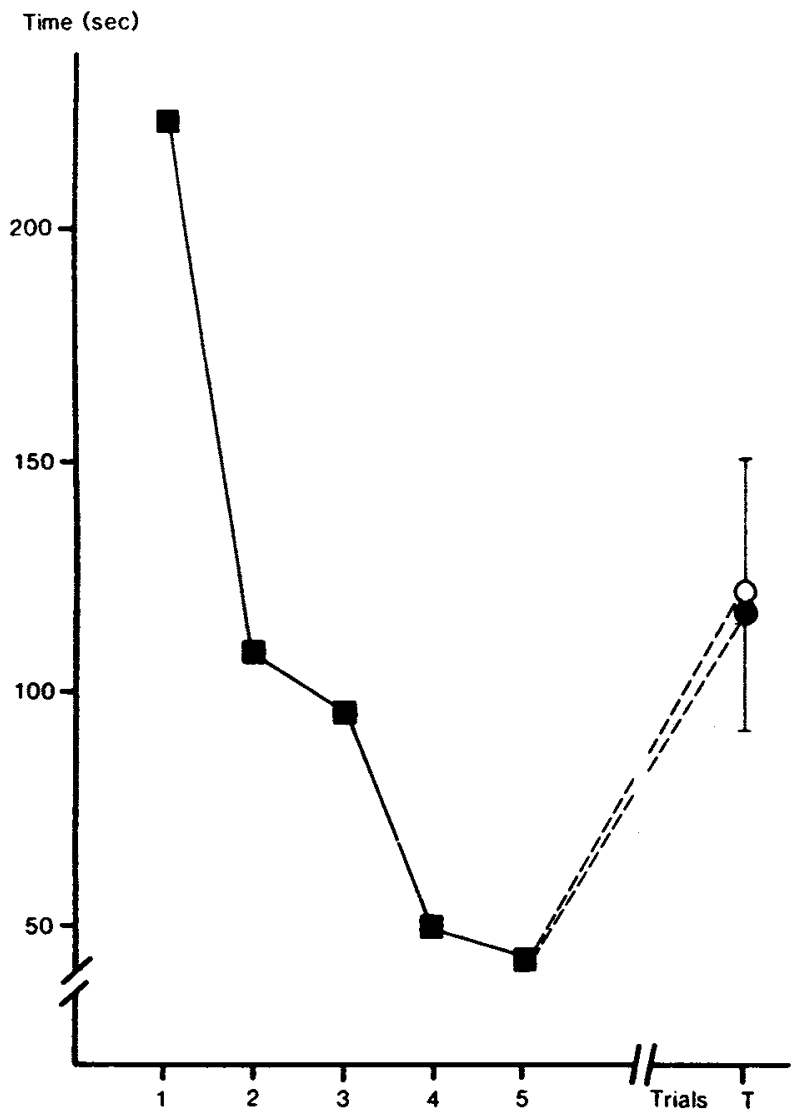

\section{EXPERIMENT 3}

The aim of this experiment was to assess the possibility of reactivating an old memory trace during paradoxical sleep. The basic experimental design was the same as that in Experiments 1 and 2, but now the cue was delivered during a PS phase which preceded the retention test.

\section{Experimental Plan and Procedure}

Forty-four rats were used as subjects. Reactivity to ETS was determined for each animal before training; the mean threshold intensity was $144 \mu \mathrm{A}$ (95 to $160 \mu \mathrm{A})$. Pretraining, training, retention interval, and testing were identical to those of Experiment 2.

On the test day, vigilance states were polygraphically monitored. The animals were divided into two groups, equalized on the basis of their performance on the last two training trials:

1. The cued rats (Group PS-C, $n=23$ ) received the cuing treatment during a PS phase which preceded testing. ETS began $20 \mathrm{sec}$ after the onset of PS. As in Experiment 2, ETS intensity was progressively increased, but was never strong enough to awaken any rat. The mean intensity of ETS was $18 \%$ below the individual thresholds assessed before training. On average, 10.2 ETSs were delivered in $80 \mathrm{sec}$ during PS.

2. The noncued rats (PS-NC, $n=21$ ) were submitted to the same manipulations as the former group except that they did not receive ETS during the PS phase that preceded the retention test.

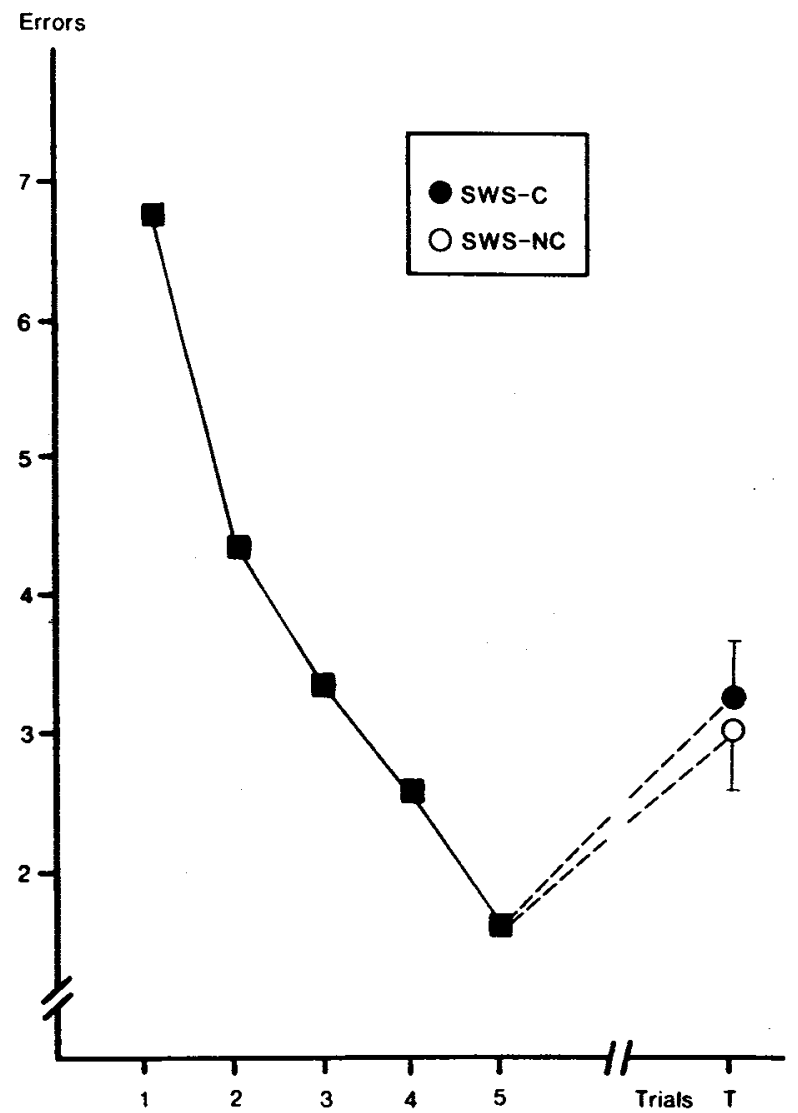

Figure 3. Mean time to run the maze (on the left) and mean errors (on the right) for combined groups during acquisition and for individual groups at retention test $( \pm S E M) 25$ days later. All animals received ETS during training. Just prior to testing, Group SWS-C received nonawakening ETS during a period of slow-wave sleep. Group SWS-NC received no ETS before testing. Note that there was no effect of pretest cuing during slow-wave sleep on retention performance, whatever performance criterion is considered. 
The two groups were tested immediately after spontaneous awakening from PS.

This experiment was run in two replications with, respectively, 24 and 20 animals in each. Since statistical comparisons showed no difference between these replications, either in acquisition or in retention performance, the data were pooled for subsequent analyses.

\section{Results and Discussion}

As in the preceding experiments, the animals showed marked improvement in performance throughout training (see Figure 4).

Delivery of ETS during pretest PS did not modify PS duration. Mean duration of the pretest PS phase was $104 \mathrm{sec}$ in the cued rats and $104.5 \mathrm{sec}$ in the noncued rats.

For each group, within-group comparisons revealed a significant performance deficit at testing as compared with that in the last training trial. Both PS-C and PS-NC animals made more errors [respectively, $F(1,22)=24.7$, $p<.001$, and $F(1,20)=4.98, p<.05$ ] and took longer to run the maze $[F(1,22)=38.39, p<.001$, and $F(1,20)$ $=16.6, p<.001]$.

However, as can be seen from Figure 4, the performance impairment at testing was more pronounced for animals cued during PS. Between-group comparisons showed that these rats (PS-C) made more errors than did the noncued rats (PS-NC) $[F(1,42)=12.55, p<.001]$. The difference between running times was not significant $[F(1,42)=2.92, p<.10]$.

Thus, the performance impairment after a long trainingto-test interval is more pronounced when a cuing treatment has been given in a PS phase before testing. As mentioned previously, this experiment was run twice, and it should be noted that twice the results were the same: the PS-cued animals made more errors than the noncued animals in the two series $[F(1,22)=5.74, p<.025$, and $F(1,18)=7.16, p<.025$, respectively]. Thus, the detrimental effect of PS cuing on retention performance is robust and reliable.

\section{EXPERIMENT 4}

After the last experiment, a question remained about the specificity of the cuing treatment. The impairment of performance by cuing could be due to nonspecific effects resulting from the introduction of a stimulus during pretest PS. The delivery of numerous earshocks might have led to disturbed sleep, even if no awakening could be detected. It could be argued that a disorganized sleep immediately
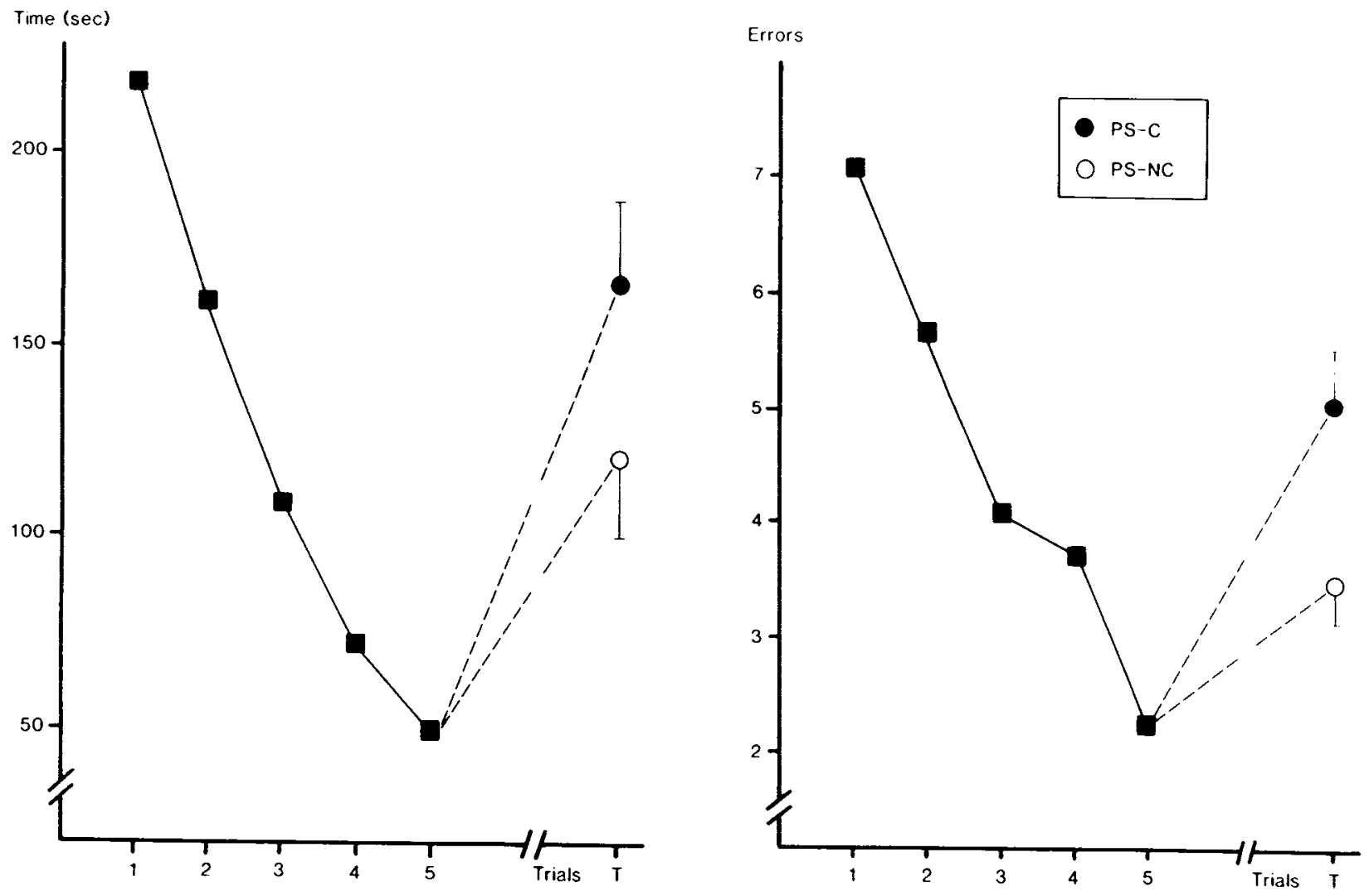

Figure 4. Mean time to run the maze (on the left) and mean errors (on the right) for combined groups during acquisition and for individual groups at retention test $( \pm S E M) 25$ days later. All animals received ETS during training. Just prior to testing, Group PS-C received nonawakening ETS during a phase of paradoxical sleep. Group PS-NC received no ETS before testing. Note the larger performance deficit as measured by errors after pretest cuing during paradoxical sleep. 
prior to testing induces poor subsequent performance. If so, we would expect that any stimulus, even a meaningless one, would likewise decrease performance when given during a PS phase before testing. The purpose of the following experiment was to see whether the same deleterious effect on retention performance could be produced when pretest earshocks had not been previously associated with the original training.

\section{Experimental Plan and Procedure}

Twenty-five rats were used as subjects. ETS behavioral thresholds were assessed for each animal before training; their mean intensity was $152 \mu \mathrm{A}$ (110 to $175 \mu \mathrm{A})$. Every aspect of the procedure was the same as in Experiment 3, except that the rats learned the maze without ETS in blind alleys. After training, the animals were divided into two groups according to the criteria described previously.

1. The cued rats (Group PS-C, $n=12$ ) received ETS in a PS phase, in the same conditions as did Group PS-C in Experiment 3. Mean intensity of ETS was $17 \%$ lower than behavioral threshold. During PS, an average of 11.5 ETSs were delivered in about $85 \mathrm{sec}$.

2. The noncued rats (Group PS-NC, $n=13$ ) did not receive ETS during the PS phase that preceded the retention trial.

\section{Results}

As shown by learning curves for both errors and run times in Figure 5, performance was quite similar to that observed in Experiment 3. This is not surprising, since in Experiment 1 there was no effect on rate of acquisition when ETS was given in the maze.

As in Experiment 3, ETS delivered during PS did not modify PS duration. Mean duration of the pretest PS phase was 110 and $102 \mathrm{sec}$ for PS-C and PS-NC, respectively.

Animals of the two groups, PS-C and PS-NC, ran more slowly and made more errors at testing than they did on the last training trial. Within-group comparisons showed this performance decrement to be significant for both errors $[F(1,11)=8.93, p<.0025$, and $F(1,12)=7.97$, $p<.025$, respectively $]$ and running times $[F(1,11)=$ $21.34, p<.001$, and $F(1,12)=14.36, p<.01]$.

By contrast with Experiment 3, the amount of forgetting in this experiment was similar in cued and noncued animals. Between-group comparisons showed no difference for either errors or run times $[F(1,23)<1$, for each index]. It can be noted that, in both groups, performance level at testing was similar to that observed in the control animals of Experiment 3, whatever performance index was considered (see Figure 5).

Thus, earshocks that have not been associated with the learning task have no effect on subsequent performance when they are given in pretest PS.
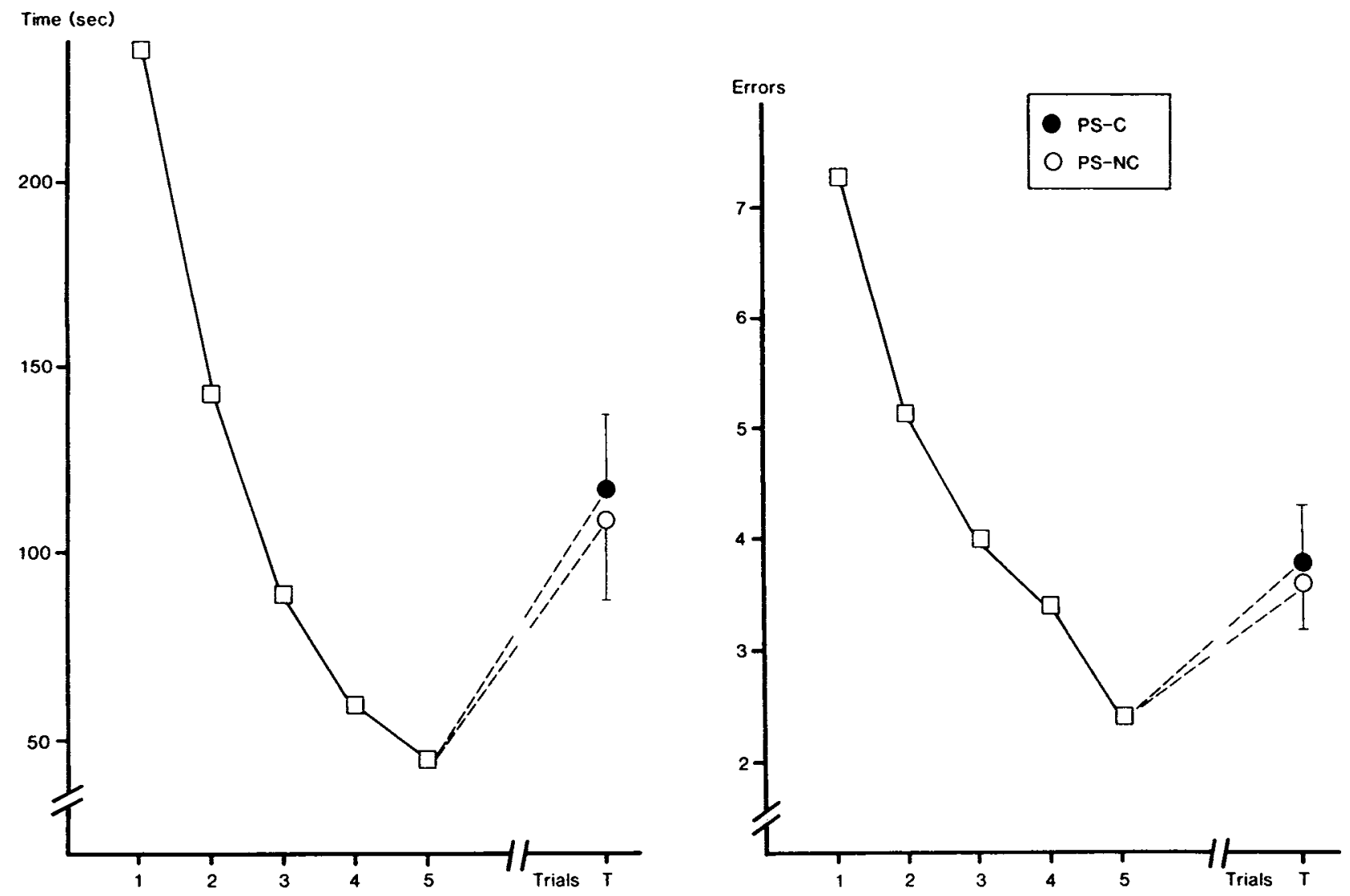

Figure 5. Mean time to run the maze (on the left) and mean errors (on the right) for combined groups during acquisition and for individual groups at retention test $( \pm S E M) 25$ days later. Neither group received ETS during training. Just prior to testing, Group PS-C received nonawakening ETS during a phase of paradoxical sleep. Group PS-NC received no ETS before testing. Note that there was no effect of pretest ETS when they had not previously been associated with training. 
Baseline performances during acquisition trials and at testing are quite similar in the present experiment and in the preceding one, which allows for comparisons between the two experiments. It appears that ETSs given in pretest PS have a detrimental effect on subsequent performance when they have been associated with the learning task (Experiment 3). On the other hand, they have no effect when they are meaningless (Experiment 4). This allows for the conclusion that the differential effect of ETS presentation in pretest PS depends upon the relevance of the ETS to the learning task.

\section{GENERAL DISCUSSION}

\section{Wakefulness}

Experiment 1 provided clear evidence of improved retention performance when pretest cuing is delivered during wakefulness. This result agrees with a whole body of experimental data that show that memory deficits arising from diverse sources can be alleviated by reminder cues (for a review, see Miller, Kasprow, \& Schachtman, 1986). These memory recoveries are generally interpreted in terms of facilitation of retrieval resulting from reactivation of the target memory triggered by cuing. Indeed, memory is viewed as a collection of attributes representing the different events noticed during learning. Cuing, by arousing an attribute, would elicit reactivation of the entire memory (Spear, 1973). Within this conceptual framework, it is evident that only a stimulus associated with the learning task is capable of giving access to the memory. The associative value of the stimulus is a determinant of its effectiveness as a reactivator. This was verified in Experiment 1. The stimulus has no effect when it has not been associated with the learning task. This rules out an interpretation in terms of a nonspecific effect of the pretest presentation of the cue. The fact that the facilitative effect of cuing is obvious only on errors and not on running time reinforces this idea.

The effectiveness of the cue raises the question of the status of this stimulus in the task. ETS cannot be considered as a discriminative stimulus for mistakes since it was given after the animal had entered a blind alley. It could be argued that ETS acts as a secondary reinforcement in the maze. This cannot be totally ruled out, but if ETS has any aversive value it is weak enough to have no detectable effect on learning performance. So, we prefer to consider ETS as an intratask cue linked to blind alleys, as could be any particular detail used to mark this part of the maze.

In this experiment, cuing produced facilitation of retention performance even though the cuing context did not correspond to the original training context. On the other hand, it has been shown (Mowrer \& Gordon, 1983) that cuing in a novel context, different from that of training, could result in poorer retention performance than if no cuing had occurred. One interpretation proposed to explain this decrease in performance is that contextual stimuli present at the time of cuing might acquire control over responding, thus diminishing the control exerted by the training context and resulting in impaired retention performance. This discrepancy with the present results is probably due to the fact that, in our experimental conditions, the cuing context was quite familiar to the animals, since it was the home cage. This context is presumably not sufficiently significant to gain control over retention performance. So, a clear facilitative effect of the nominal stimulus by itself can be observed, even though the context is changed.

Furthermore, the results of both this experiment and the pilot study corroborate those of previous experiments that have used a similar long-delay retention paradigm (Deweer \& Sara, 1984; Deweer et al., 1980; Sara, Deweer, \& Hars, 1980). By demonstrating that pretest cuing can alleviate forgetting, they support the idea that partial forgetting represents a retrieval failure (Spear, 1973) rather than a decay of the trace (Gleitman, 1971). The main difference between the present experiment and the earlier ones lies in the nature of the reminder, since we used exposure to the training context as the cuing treatment in the earlier ones. Thus, a stimulus that occurs only episodically during training seems to be as effective as background stimuli that are permanently present. Indeed, despite its intermittency, ETS was closely associated with the target memory, since it was strictly absent outside the learning trials. Moreover, our rats had never experienced ETS, and although its intensity was weak, its novelty must imply salience. This salience can facilitate ETS integration as a memory component and consequently can improve the power of this stimulus as a reminder cue.

\section{Slow-Wave Sleep}

Experiment 2 showed that pretest cuing in SWS has no effect on retention performance. Several alternatives can be proposed concerning this lack of effect. First, it can be argued that a nonawakening external stimulus is not able to "penetrate" and to be processed because of the isolation of the organism from the environment during sleep. Although this cannot be precluded, since we had no direct indication of stimulus perception during sleep, several other studies (Ciancia, Trigona-Leisinger, \& Bloch, 1980; Halperin \& Iorio, 1981; Van Twyver \& Garrett, 1972) have shown that the ability of sleeping organisms to detect external information is enhanced when an already significant stimulus is used. Moreover, some data have indicated that a signal can be recognized, and even that some components of a conditioned response can be expressed, in SWS (Gauthier, Hammon, \& Gottesmann, 1986; Sasaki \& Yoshii, 1984). Second, it may be that a reactivation cannot spread in SWS or, if it can, that its effects cannot transfer to wakefulness. On the other hand, we have previously shown that a cuing procedure can be used in SWS and can alter subsequent performance in wakefulness (Hars \& Hennevin, 1987). However, this effect has been observed with repeated cuing in several SWS phases, whereas here the cue is delivered in only one SWS phase. So our present experimental conditions may not be ideal for obtaining an effect of cuing during SWS: 
either cuing is insufficient to reach reactivation threshold or memory reactivation is not complete enough to have any tangible effect on subsequent performance.

As with all negative results, one cannot conclude from this lack of cuing efficacy that memory reactivation is impossible in slow-wave sleep.

\section{Paradoxical Sleep}

The results of Experiment 3 showed that pretest cuing during a PS phase impairs retention performance. This deficit cannot be due to the presence of PS before testing, since, in the control group, retention was also evaluated after a PS phase. Furthermore, Experiment 4 shows that the stimulus used as the cue must have been associated with the learning task in order to have an effect. This precludes any explanation in terms of a nonspecific effect of the stimulus. It is only when the stimulus is an attribute of the target memory that it can reach this memory to trigger its reactivation.

Such an interpretation in terms of memory reactivation in PS is consistent with our previous results relative to recent memories. Indeed, in two kinds of experiments, we have shown that a memory trace can be modulated during PS. First, we found that it was possible for retention to be facilitated by electrical stimulation of the mesencephalic reticular formation administered during postlearning PS, just as it can be when applied just after learning. This suggests that the memory is in an active state during PS, as it is just after acquisition in wakefulness (Hennevin et al., 1989). Second, we found that intersession cuing was effective when delivered during PS, which suggests that a memory trace remains accessible to a reminder in PS (Hars et al., 1985). Taken together, these results argue strongly for the possibility of memory reactivation in PS.

The puzzling point in the present results is the facilitative effect of pretest cuing when it is given in wakefulness and the deleterious effect when it is given in PS. Although it is easy to understand that target memory reactivation can facilitate its subsequent expression, the inverse effect can, at first glance, seem paradoxical. However, there are many reports in the literature that indicate that cuing procedures result in a retention deficit when a treatment, which has amnestic effects when delivered just after learning, is administered during a cuing episode, even a long time after learning (Devietti \& Kirkpatrick, 1976; Judge \& Quartermain, 1982). This is also true when the cuing episode occurs in an irrelevant context (Mowrer $\&$ Gordon, 1983). From these observations, two proposals can be put forward to account for the present effect of cuing in PS.

The first consists of reconsidering the interpretation generally advanced to account for the reestablished memory lability that accompanies cuing during wakefulness. The cue, by restoring the trace into an active state, makes it sensitive to either facilitative or disruptive memory modulators. In the present case, this hypothesis would lead one to consider PS, or one of its components, to be an amnesic agent. This interpretation fits well with Crick's speculations (Crick \& Mitchison, 1983) about the role of PS in memory, that is, that PS serves to erase information stored in wakefulness. This assumption is based mainly on the commonly observed forgetting of dreams. For Crick and Mitchison, forgetting would result from a PS's "reverse learning mechanism" which weakens memory traces. But allocation of such a role to PS is not tenable in light of the large amount of existing data that shows that postlearning PS has a beneficial effect on retention (Bloch, Hennevin, \& Leconte, 1979; McGrath \& Cohen, 1978; Pearlman, 1979; Smith, 1985). Thus, within this interpretative framework, it is reasonable to consider that it is not PS itself which has a deleterious effect. An alternative is to propose that the deficit may be due to the break caused by the change of vigilance state at the end of the PS phase. Awakening, by occurring when the memory trace is still active, could have a disruptive effect and serve as a source of immediate retrieval failure.

The second possibility is that the trace is altered by being evoked in conditions that differ too much from those present at its formation. When reactivated, a trace becomes "malleable" (Gordon, 1983) and can be modified by incorporation of new information present when the trace is reactivated. For example, Mowrer and Gordon (1983) have shown that cue reactivation that takes place in a novel and nonpertinent context (i.e., different from that of acquisition and restitution) impairs retention performance. Obviously, state of vigilance must be an important aspect of the internal context, and internal context provided by PS could be too different from that of wakefulness associated with training. Moreover, some irrelevant information could be running on in PS 1 month after learning and could interfere with the reactivated old memory. These interactions may produce some modifications of the original training memory, either by the addition of a new contextual representation or by alteration of any of its existing components. Such an evolution of the content of the original memory should decrease its retrievability.

Another puzzling point is the facilitative effect of intertrial reactivation in PS obtained in a previous experiment (Hars et al., 1985) and the disruption observed here. Of course, comparisons are limited by the many disparities between the two experiments. In the former, the learning task was active avoidance, the cue used was the conditioned stimulus (CS), the intersession cuing occurred in postlearning PS, it was repeated over six consecutive PS phases, and its effect was evaluated $24 \mathrm{~h}$ later. One or many of these procedural differences could be the source of the differential effect of cuing in the two cases. For instance, in the present experiment, the cuing procedure was given in only one PS phase and the cue was not the nominal CS. These conditions could permit only an incomplete reactivation of the trace, which may be insufficient to produce a beneficial effect. Nevertheless, the question remains as to whether a partial reactivation can have a deleterious effect on subsequent performance. 
Another possibility is that an old memory and a new one may be sensitive in different ways to reactivation in PS. Data from Mactutus and others (Mactutus, Ferek, George, \& Riccio, 1982; Mactutus, Riccio, \& Ferek, 1979; Maki, $1986)$ reveal that a memory has greater vulnerability when it is reactivated than when it is a new one, since a mild amnesic treatment disrupts a reactivated trace but spares a new trace. So, reprocessing in PS could be beneficial to a growing memory, whereas reactivation in PS could alter a partially forgotten memory. Whatever the mechanism implicated, a process that allowed for a weakening of old memories and a strengthening of new ones would have an evident adaptative function. Such an assumption is only speculative; further research that is specifically designed to compare the effect of reactivation in PS on an old memory and a new one is needed. Nevertheless, this result, which suggests the lessening of an old memory trace when reactivated in PS, is important in terms of the theoretical problem of the interaction between the "printing" of new information and the preexisting contents of the memory store.

\section{Conclusion}

The present data show that the memory reactivation effect depends upon the vigilance state in which it takes place. This general statement agrees with our previous results concerning the effects of new memory reactivation in wakefulness and different stages of sleep (Hars \& Hennevin, 1987; Hars et al., 1985). Taken together, all these data support the idea that dynamic memory processing is not confined to wakefulness-that it can also take place in sleep.

\section{REFERENCES}

Bloch, V., Hennevin, E., \& Leconte, P. (1979). Relationship between paradoxical sleep and memory processes. In M. A. Brazier (Eds.), Brain mechanisms in memory and learning from the single neuron to man. New York: Raven Press.

Ciancia, F., Trigona-Leisinger, M. C., \& Bloch, V. (1980). Etude, chez le chat, de la réactivité aux stimulus auditifs signifiants en fonction des niveaux de vigilance. Archives Italiennes de Biologie, 118, 375-385

Crick, F., \& Mitchison, G. (1983). The function of dream sleep. $\mathrm{Na}$ ture, 304, 111-114

Dekeyne, A., Deweer, B., \& SARA, S. (1987). Background stimuli as a reminder after spontaneous forgetting: Potentiation by stimulation of the mesencephalic reticular formation. Psychobiology, 15, 161-166.

DEVIETTI, T. L., \& KIRKPaTRICK, B. R. (1976). The amnesia gradient: Inadequate as evidence for a memory consolidation process. Science, 194, 438-440.

DEWEER, B., \& SARA, S. J. (1984). Background stimuli as a reminder after spontaneous forgetting: Role of duration of cuing and cuing-test interval. Animal Learning \& Behavior, 12, 238-247.

Deweer, B., Sara, S. J., \& Hars, B. (1980). Contextual cues and memory retrieval in rats: Alleviation of forgetting by a pretest exposure to background stimuli. Animal Learning \& Behavior, 8, 265-272.

Fishbein, W., \& Gutwein, B. M. (1977). Paradoxical sleep and memory storage processes. Behavioral Biology, 19, 425-464
Gatti, S. V., Pais, N., \& Weeks, J. R. (1975). Effect of reinstatement procedure on retention of differential appetitive responding. Bulletin of the Psychonomic Society, 6, 57-60.

Gauthier, P., Hammon, J. F., Gottesmann, C. (1986). Etude de la variation contingente négative et des composantes tardives des potentiels évoqués au cours du sommeil chez l'homme. Revue d'EEG et de Neurophysiologie Clinique, 15, 323-330.

Gisquet-Verrier, P., Dekeyne, A., \& Alexinsky, T. (1989). Differential effect of retrieval cues over time: Evidence for time reorganization of memory. Animal Learning \& Behavior, 17, 394-408.

Gleitman, H. (1971). Forgetting of long-term memories in animals. In W. K. Honig \& P. H. R. James (Eds.), Animal memory. New York: Academic Press

GoRdon, W. C. (1983). Malleability of memory in animals. In R. L. Mellgren (Ed.), Animal cognition \& behavior. Amsterdam: NorthHolland.

HALPERIN, J. M., \& IoRIo, L. C. (1981). Responsivity of rats to neutral and danger-signalling stimuli during sleep. Behavioral \& Neural Biology, 33, 213-219.

Hars, B., Hennevin, E. (1987). Impairment of leaming by cueing during postlearning slow-wave sleep in rats. Neuroscience Letters, 79, 290-294.

Hars, B., Hennevin, E., \& Pasques, P. (1985). Improvement of learning by cueing during postlearning paradoxical sleep. Behavioural Brain Research, 18, 241-250.

HEINE, R. (1914). Über wiedererkennen und ruckwirkende Hemmung. Zeitschrift für Psychologie und Physiologie der Sinnesorgane, 68. 161-236.

Hennevin, E., \& Hars, B. (1985). Post-learning paradoxical sleep: A critical period when new memory is reactivated? In B. E. Will, P. Schmitt, J. C. Dalrymple-Alford (Eds.), Brain plasticity, learning and memory. New York: Plenum.

Hennevin, E., Hars, B., Bloch, V. 1989). Improvement of learning by mesencephalic reticular stimulation during postleaming paradoxical sleep. Behavioral \& Neural Biology, 51, 291-306.

Hennevin, E., \& Leconte, P. (1977). Etude des relations entre le sommeil paradoxal et les processus d'acquisition. Physiology \& Behavior, 18, 307-319.

Jenkins, J. G., \& Dallenbach, K. M. (1924). Obliviscence during sleep and waking. American Joumal of Psychology, 35, 605-612.

Judge, M. E., \& QuARTERmain, D. (1982). Characteristics of retrograde amnesia following reactivation of memory in mice. Physiology \& Behavior, 28, 585-590.

LEWIS, D. J. (1969). Sources of experimental amnesia. Psychological Review, 76, 461-472

LEWIS, D. J. (1979). Psychobiology of active and inactive memory. Psychological Bulletin, 86, 1054-1083.

Mactutus, C. F., Ferek, J. M., George, C. A., \& Riccio, D. C. (1982). Hypothermia-induced amnesia for newly acquired and old reactivated memories: Commonalities and distinctions. Physiological Psychology, 10, 79-85.

Mactutus, C. F., Riccio, D. C., \& FereK, J. M. (1979). Retrograde amnesia for old (reactivated) memory: Some anomalous characteristics. Science, 204, 1319-1320.

MAKI, W. S. (1986). Distinguishing between new and used traces: Differential effects of electroconvulsive shock on memories for places presented and places passed. Quarterly Journal of Experimental Psychology, 38B, 397-423.

Mandai, O., Guerrien, P., Sockeel, P., Dujardin, K. \& LECONTE, P. (1989). REM sleep modifications following a morse code learning session in humans. Physiology \& Behavior, 46, 639-642.

MCGrath, M. J., \& CoHEN, D. B. (1978). REM sleep facilitation of adaptative waking behavior: A review of the literature. Psychological Bulletin, 85, 24-57.

Miller, R. R., Kasprow, W. S., \& Schachtman, T. R. (1969). Retrieval variability: Sources and consequences. American Journal of Psychology, 99, 145-218.

MOWRER, R. R., \& GoRDON, W. C. (1983). Effects of cuing in an "irrelevant" context. Animal Learning \& Behavior, 11, 401-406. 
Pearlman, C. A. (1979). REM sleep and information processing: Evidence from animal studies. Neuroscience \& Biobehavioral Reviews, 3, 57-68.

Sara, S. J., Deweer, B., \& Hars, B. (1980). Reticular stimulation facilitates retrieval of a "forgotten" maze habit. Neuroscience Letters, 18, $211-217$.

SASAKI, H., YOSHII, N. (1984). Conditioned responses in the visual cortex of dogs during sleep. Electroencephalography \& Clinical Neurophysiology, 58, 448-456.

SMITH, C. (1985). Sleep states and learning: A review of the animal literature. Neuroscience Behavioral Review, 9, 157-168.

Spear, N. E. (1973). Retrieval of memory in animals. Psychological Review, 80, 163-194.
SPEAR, N. E. (1978). The processing of memories: Forgetting and retention. Hillsdale, NJ: Erlbaum.

Timo-Iaria, C., Negrad, N. Schmidek, W. R., Hoshino, K., Menezes, C. E., DA Rocha, T. L. (1970). Phases and states of sleep in the rat. Physiology \& Behavior, 5, 1057-1062.

VAN ORMER, E. B. (1932). Retention after intervals of sleep and of waking. Archives of Psychology, 137, 5-49.

VAN Twyver, H., Garrett, N. (1972). Arousal threshold in the rat determined by "meaningful" stimuli. Behavioral Biology, 7, 205-215.

(Manuscript received December 20, 1989; revision accepted for publication May 16, 1990.) 